COMECHINGONIA. Revista de Arqueología. Vol. 22, n² 1. Primer semestre de 2018. ISSN: 0326-7911

COMITÉ EDITORIAL

DIRECTOR

SEBASTIÁN PASTOR (CITCA-CONICET-Catamarca)

CO-EDITORA

ANDREA RECALDE (CEH-IEH-CONICET-UNC-Córdoba)

CONSEJO ASESOR

JESÚS ADÁNEZ PAVÓN (UNIVERSIDAD COMPLUTENSE-Madrid)

J. ROBERTO BÁRCENA (INCIHUSA-CONICET-UNCU-Mendoza)

LUIS F. BATE (ENAH-México)

LUIS BORRERO (IMHICIHU-CONICET-Buenos Aires)

FELIPE CRIADO BOADO (INCIPIT-CSIC-Santiago de Compostela)

LEONARDO GARCÍA SANJÚAN (U. DE SEVILLA-Sevilla)

GUILLERMO MENGONI GOÑALONS (ICA-UBA-CONICET-Buenos Aires)

AXEL NIELSEN (INAPL-CONICET-Buenos Aires)

GUSTAVO POLITIS (INCUAPA-CONICET-UNCPB-Olavarría)

MYRIAM TARRAGÓ (M. ETNOGRÁFICO-UBA-CONICET-Buenos Aires)

HUGO YACOBACCIO (ICA-UBA-CONICET-Buenos Aires)

EVALUADORES PARA ESTE NÚMERO

Federico Bobillo (ISES-CONICET); María F. Bugliani (IDECU-UBA-CONICET); Rafael

Curtoni (INCUAPA-CONICET-UNCPBA); Violeta Di Prado (FCNyM-UNLP-CONICET);

Anabel Feely (IDECU-UBA-CONICET); Fernanda Falabella (Dpto. de Antropología, Universidad de Chile); Emilia Iucci (FCNyM-UNLP-CONICET ); Jimena Oria (CADICCONICET); María C. Páez (CONICET-FCNyM-UNLP); Valeria Palamarzuck (IDECUUBA-CONICET); Rafael Paunero (FCNyM-UNLP); José Porto López (LARBO-UNMDP); Cristina Prieto Olavarría (IANIGLA-CONICET); Federico Restifo (CONICET-FFyH-UBA);

Camila Riera Soto (Universidad Católica del Norte, Chile); Julián Salazar (CEH-IEHCONICET-UNC); M. Lorena Sanhueza Riquelme (Dpto. de Antropología, Universidad de Chile); Marina Sprovieri (CONICET-FCNyM-UNLP); Emily Stovel (El Zócalo Plaza Visitor and Event Center, Sandoval County Office of Economic Development, Bernalillo); Nuria Sugrañes (IANIGLA-CONICET); Veronika Szilágyi (Nuclear Analysis and Radiography Department, Centre for Energy Research, Hungarian Academy of Sciences); Cristian Vitry (UNSa); Verónica Williams (IDECU-UBA-CONICET); Federico Wynveldt (CONICETFCNyM-UNLP); M. Amalia Zaburlin (CREA-FHyCS-UNJu).

Dirección postal: Miguel C. del Corro 308, (5000) Córdoba - Argentina

Correo electrónico: revistacomechingonia@gmail.com

Web: http//www.comechingonia.com 

COMECHINGONIA. Revista de Arqueología. Vol. 22, n 1. Primer semestre de 2018. ISSN: 0326-7911

\section{Índice}

Presentación

Dossier: "Problemáticas y abordajes metodológicos en los estudios petrográficos de cerámica arqueológica".

1. Presentación.

Por: Lucas Pereyra Domingorena y Verónica Puente

2. Un volcán en la cerámica: indicios de producción en el altiplano de Isluga,

Tarapacá-Chile.

Por: Isabelle Druc y Mauricio Uribe Rodríguez

3. Una puesta al día sobre la variación petrográfica y química del estilo cerámico Inca Pacajes.

Por: Nicolás Larcher y M. Beatriz Cremonte

4. Elecciones tecnológicas y prácticas alfareras en el tambo Costa de Reyes $\mathrm{N}^{\mathrm{o}}$

5 (Tinogasta, Catamarca): aproximaciones petrográficas.

Por: Sergio Vera y Guillermo de la Fuente

5. Tecnología cerámica a través de la petrografía en la Quebrada de Miriguaca (Antofagasta de la Sierra, Catamarca) durante el Período Tardío.

Por: Leticia Gasparotti

6. Nuevas evidencias en relación a la selección de materiales cerámicos para

formar parte de los contextos funerarios. PP9-III y PP13-I como casos de estudio (ca. 1330-1000 AP) (Antofagasta de la Sierra, Catamarca).

Por: Vanesa Juárez

7. Prácticas de manufactura cerámica en el oeste riojano durante el Período

Tardío (ca. siglos XIII-XVII AD). Aportes desde los estudios petrográficos sobre el estilo Sanagasta/ Angualasto de la Tambería de Guandacol.

Por: Sebastián Carosio

8. Evidencias arqueológicas de los siglos VII a XII AD en el asentamiento de Moreta (Puna de Jujuy, Argentina).

Por: M. Josefina Pérez Pieroni y Carlos Angiorama

9. Una aproximación petrográfica a los modos de hacer y la circulación alfarera en el Área Valliserrana del Noroeste Argentino durante los últimos siglos del primer milenio D.C.

Por: Lucas Pereyra Domingorena y Verónica Puente

\section{Artículos}

1. Nuevos datos en relación a la arqueología del valle de Lerma, provincia de

Salta, Argentina.

Por: Cecilia Mercuri

2. Aplicación de los drones en diversos contextos arqueológicos y en casos de reconstrucción geoarqueológica.

Por: M. Marta Sampietro Vattuone y José Peña Monné 
3. Ritualidad y espacialidad andina a través de enfoques etnográficos y arqueológicos. Confluencias cosmogónico-ontológicas en la constitución de espacios sagrados entre El Shincal de Quimivil y ceremonias actuales de origen cusqueño.

Por: Marco Giovannetti

4. El paisaje rupestre de Cerro Colorado (provincia de Córdoba, Argentina): detectando otros sentidos, otras corporalidades.

Por: Luis Tissera 
COMECHINGONIA. Revista de Arqueología. Vol. 22, n 1. Primer semestre de 2018. ISSN: 0326-7911

\title{
TECNOLOGÍA CERÁMICA A TRAVÉS DE LA PETROGRAFÍA EN LA QUEBRADA DE MIRIGUACA (ANTOFAGASTA DE LA SIERRA, CATAMARCA) DURANTE EL PERÍODO TARDÍO
}

\section{CERAMIC TECHNOLOGY THROUGH PETROGRAPHY IN MIRIGUACA RAVINE (ANTOFAGASTA DE LA SIERRA, CATAMARCA) DURING THE LATE PERIOD}

\author{
Leticia Gasparotti ${ }^{1}$ \\ 1 CITCA-CONICET; Escuela de Arqueología-UNCa, Prado 366, \\ (4700) Catamarca, Argentina, lgasparotti@arqueo.unca.edu.ar
}

Presentado: 11/04/2017 - Aceptado: 06/11/2017

\section{Resumen}

Este trabajo es el resultado de las investigaciones realizadas sobre el material cerámico del sitio Corral Alto (Antofagasta de la Sierra, Provincia de Catamarca). A través del estudio petrográfico de la alfarería proveniente de este sitio se presenta una primera aproximación de los modos de hacer cerámica durante el Período Tardío en la Quebrada del río Miriguaca. A lo largo de este trabajo se buscó determinar la composición mineralógica de las pastas cerámicas, examinar la relación de estas con las características geológicas locales, e indagar en las diversas elecciones tecnológicas y los modos de hacer particulares de este conjunto, pensando en las vasijas cerámicas como objeto que ensambla y reúne materiales, personas y lugares en extensas redes de relaciones. Se consideró un universo de estudio de 2171 tiestos cerámicos separados en 16 grupos o familias de fragmentos. Partiendo de estos grupos, en esta oportunidad, 37 muestras se analizaron petrográficamente. De este modo, se pudieron establecer algunas elecciones tecnológicas de los alfareros que vinculan la cerámica de Corral Alto con una producción local, no estandarizada y flexible. La información obtenida permitió repensar las relaciones entre estas prácticas alfareras y aquellas llevadas a cabo en diferentes sectores de la microrregión

Palabras clave: pastas cerámicas, elecciones tecnológicas, modos de hacer, Puna Meridional

\begin{abstract}
This paper is the result of the research performed on ceramic material from the site Corral Alto (Antofagasta de la Sierra, Catamarca). Through the petrographic study of the pottery from this site we tried to present a first approximation of the ways of making ceramics during the Late Period in the gorge of the Miriguaca River. Throughout this work, it was sought to determine the mineralogical
\end{abstract}


composition of ceramic pastes, to examine their relationship with local geological characteristics, and to investigate the technological choices and particular ways of doing, thinking about ceramics vessels as an object that assembles and gathers materials, people and places in extensive networks that give meaning. A study universe of 2171 ceramic fragments separated into 16 groups or families of fragments was considered. Starting from these groups, in this opportunity, 37 samples were analyzed petrographically. In this way, it was possible to establish certain characteristics of the technological choices made by the potters, what link the pottery of Corral Alto with a local, non-standardized and flexible production. The information obtained allowed to rethink the relations between these pottery practices and those carried out in other sectors of the microregion.

Keywords: ceramic pastes, technological choices, ways of doing, Southern Puna

\section{Introducción}

En este trabajo se presentan los primeros resultados de las investigaciones realizadas sobre el material cerámico procedente del sitio Corral Alto, a través de las cuales se intenta aportar a la comprensión de los procesos sociales ocurridos durante el período Tardío (ca. 1000-500 años AP) en la quebrada del río Miriguaca (Antofagasta de la Sierra, Puna Meridional Argentina). Para abordar esta problemática se recurre a la evidencia proporcionada por el material cerámico recuperado del sitio Corral Alto, examinando las características de la producción cerámica y sus implicancias a nivel social. En este sentido, este sitio permite indagar en la complejidad social creciente que se habría dado en este momento en la microrregión 1 .

La micorregión de Antofagasta de la Sierra se encuentra dentro del departamento homónimo, ubicado al noroeste de la provincia de Catamarca (Tab 1). Se define sobre la base de la red hidrográfica endorreica de la cuenca del río Punilla, alimentada y regulada por vertientes de régimen permanente. Entre sus tributarios se encuentran los río Miriguaca, Las Pitas, Ilanco, Los Colorados, Mojones, Toconquis y el arroyo de Curuto (Olivera et al. 2004; Tchilinguirian y Olivera 2010).

Hasta el momento, la mayor cantidad de trabajos arqueológicos referidos al Tardío se han concentrado en el sector denominado "Fondo de Cuenca" 2 y en grandes sitios habitacionales como La Alumbrera o extensas áreas agrícolas como Bajo del Coypar. En este sentido, las investigaciones generaron una cartografía social construida sobre la base de la distribución diferencial de las actividades productivas y la densidad poblacional (Cohen 2011). Este modelo, implicaría la existencia de una élite con poder centralizado sobre la producción, circulación de bienes e intercambio de los mismos, que estaría ubicada en el 
sector más bajo de la cuenca, extendiendo su control sobre el resto de la población localizada en los otros sectores.

Sin embargo, este paisaje social podría haberse generado debido al alto desfasaje en el desarrollo de las investigaciones a lo largo del tiempo, que privilegiaron históricamente al sector de fondo de cuenca dejando prácticamente de lado la exploración de los cursos inferior y medio de las quebradas subsidiarias, situación que desde hace un tiempo ha comenzado a cambiar gracias a los trabajos de distintos investigadores. Es por ello que los sectores intermedios de las quebradas han tomado relevancia para la comprensión de la complejidad social que se dio durante el Tardío en la microrregión (Escola et al. 2015). En esta oportunidad se abordan específicamente las evidencias existentes en la quebrada del río Miriguaca.

Allí, a 3680 msnm se localiza el sitio Corral Alto. Dispuesto sobre una terraza sobreelevada, delimitada y en parte conformada por un farallón de ignimbritas, característico de estos sectores, el sitio cuenta con una vista privilegiada de la vega y todo su entorno, con un amplio control visual de sus alrededores. Es probable que su acceso haya sido restringido debido a su localización particular, su escasa visibilidad desde la vega y la pendiente pronunciada que hay que superar para llegar hasta él.

Dentro del sitio se diferencian dos grandes sectores, cada uno compuesto por un número escaso de recintos y diferenciados por una marcada pendiente entre ellos (Figura 2). El primero, por el cual se accede al sitio, es el sector SO. Presenta un muro de pirca seca que delimita un espacio semicircular de grandes dimensiones (a modo de "patio") con una superficie de alrededor de $360 \mathrm{~m}^{2}$., dispuesto contra el farallón ignimbrítico. En su interior pueden diferenciarse un conjunto de estructuras circulares de pirca seca que dividen este gran espacio (tres contra la pared del farallón y una adosada al patio) (Escola et al. 2013).

Siguiendo en dirección NE, un extenso muro de pirca seca guía la circulación en el sitio y obliga a transitarlo por un estrecho pasillo, que conduce al segundo sector (NE), ubicado en la parte más baja, donde se halla un conjunto de estructuras rectangulares de pirca y muros elevados.

En este sector fueron excavadas dos estructuras (E1 y E2). La estructura E1 fue excavada en su totalidad. Se registraron dos niveles arqueológicos con una potencia de aproximadamente $15-20 \mathrm{~cm}$. La mayor parte de los hallazgos corresponden al nivel dos (Grant y Escola 2015). 


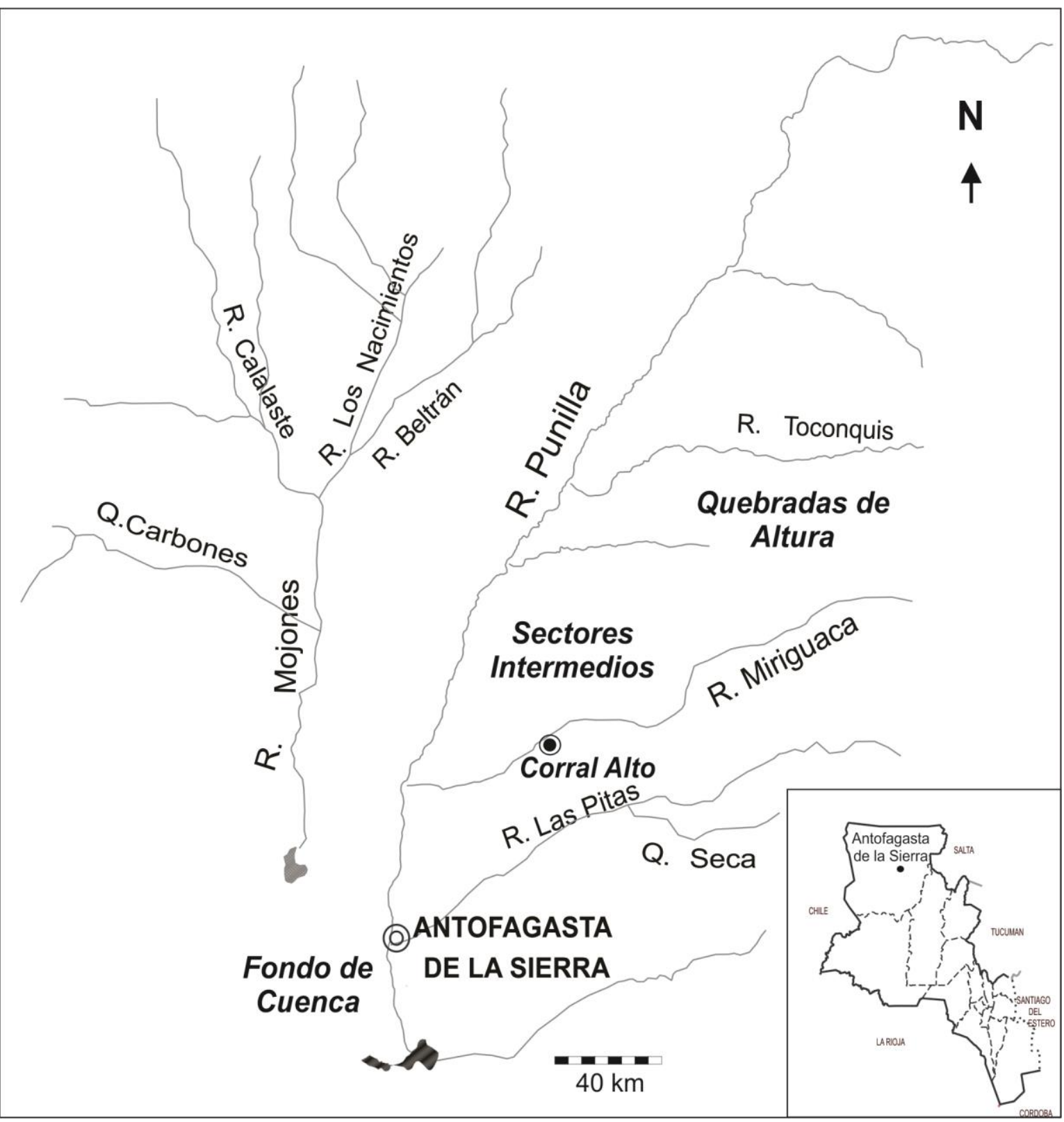

Figura 1. Mapa de la microrregión de Antofagasta de la Sierra y ubicación del sitio Corral Alto.

Las excavaciones permitieron la obtención de cuatro fechados radiocarbónicos que ubican la ocupación del sitio durante el Período Tardío. 


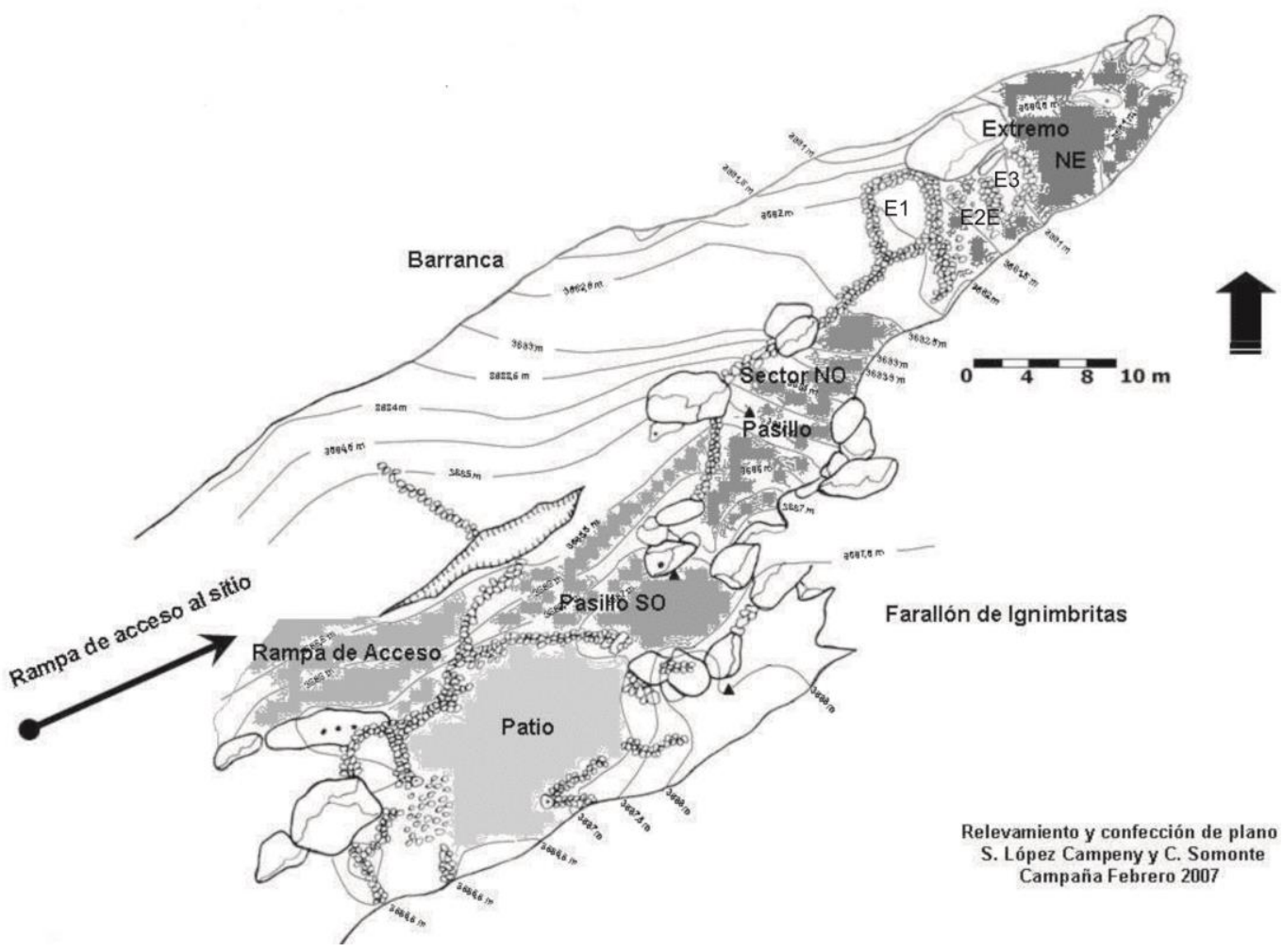

Figura 2. Planialtimetría de Corral Alto.

\begin{tabular}{|c|c|c|c|c|c|c|}
\hline $\begin{array}{c}\text { Fecha } \\
\text { AP. }\end{array}$ & $\begin{array}{c}\text { Años } \\
\text { calibrados AP } \\
(95 \% \\
\text { probabilidad) }\end{array}$ & $\begin{array}{l}\text { Años } \\
\text { Cal. } \\
\text { AP }\end{array}$ & Material & Procedencia & $\begin{array}{c}\text { Código } \\
\text { de } \\
\text { muestra }\end{array}$ & $\begin{array}{c}\text { Ref. } \\
\text { bibliográfica }\end{array}$ \\
\hline $720 \pm 40$ & $670-564$ & 665 & $\begin{array}{c}\text { AMS } \\
\text { endocarpo } \\
\text { de chañar }\end{array}$ & E 1 & $\begin{array}{l}\text { Ua- } \\
33241\end{array}$ & $\begin{array}{l}\text { Escola et. al. } \\
2013\end{array}$ \\
\hline $660 \pm 60$ & $670-529$ & 601 & $\begin{array}{l}\text { Carbón } \\
\text { vegetal }\end{array}$ & E 1 & LP- 1986 & $\begin{array}{l}\text { Gasparotti y } \\
\text { Escola } 2012\end{array}$ \\
\hline $860 \pm 60$ & $905-656$ & 744 & $\begin{array}{l}\text { Carbón } \\
\text { vegetal }\end{array}$ & E 2 & LP- 2535 & $\begin{array}{c}\text { Escola et. al. } \\
2015\end{array}$ \\
\hline $970 \pm 80$ & $963-685$ & 837 & $\begin{array}{l}\text { Carbón } \\
\text { vegetal }\end{array}$ & E 2 & LP- 3087 & $\begin{array}{c}\text { Pérez y } \\
\text { Gasparotti } \\
2016\end{array}$ \\
\hline
\end{tabular}

Tabla 1. Fechados radiocarbónicos del sitio y su calibración con $2 \sigma$ realizada con el software Oxcal v. 4.1 (Bronk Ramsey 2001) y utilizando la curva ShCal04 (McCormac et al. 2004, en Grana 2013). 
Las actividades que se llevaron a cabo en Corral Alto pudieron ser inferidas a través de los estudios realizados sobre el material faunístico, lítico y cerámico. Por un lado, los restos óseos faunísticos recuperados permitieron determinar que el consumo de camélidos fue dominante en Corral Alto, tanto silvestres como domésticos. Al mismo tiempo, la alta diversidad de elementos esqueletarios registrados es la esperable en bases residenciales con una alta estabilidad ocupacional (Grant 2014; Grant y Escola 2015). Estos estudios permitieron plantear que en el curso intermedio de la quebrada se habría mantenido una economía agropastoril diversificada, que combinaría la caza de camélidos silvestres con la crianza de pequeños rebaños de llamas (Grant y Escola 2015).

Por otra parte, los estudios del material lítico concluyeron que existió una elevada presencia de instrumentos asociados a actividades extractivas, en relación con otros conjuntos contemporáneos. Específicamente llama la atención el alto porcentaje de puntas de proyectiles y preformas observadas. Se ha planteado que esta situación podría estar relacionada con las actividades de caza de camélidos silvestres que se reflejan en los restos óseos faunísticos, o bien tener vinculación con alguna situación de conflicto o tensión social que se haya dado en ese momento. El material lítico también posibilitó identificar numerosos grupos instrumentales, especialmente relacionados con la molienda, y asociados a actividades de procesamiento/consumo lo que concuerda con lo esperado para una base residencial. Además, a través del estudio de las materias primas líticas, se propone la existencia de una mayor movilidad de los habitantes de Corral Alto dentro de la cuenca, en comparación con sus contemporáneos del fondo de cuenca ${ }^{1}$, debido a la identificación de materias primas provenientes de los distintos microambientes (Elías 2010).

Finalmente, y relacionado con este trabajo en particular, el material cerámico, analizado en trabajos previos, ha dejado entrever que este conjunto sería un repertorio pensado para el almacenamiento de productos secos, granos posiblemente, aunque no siendo esta una actividad excluyente (Gasparotti y Escola 2012). En general, las vasijas de Corral Alto podrían denominarse domésticas y de factura tosca $u$ ordinaria, caracterizadas principalmente por las grandes ollas de paredes gruesas y difíciles de transportar por su peso y dimensiones, identificadas a partir del registro fragmentario. Además, muchas de las ollas reconstruidas tienen bocas de gran diámetro, que indicarían una fácil accesibilidad al contenido de los recipientes. El conjunto presenta en general muy escasas evidencias de exposición al fuego, calcinamiento o desgaste excesivo (Gasparotti y Escola 2012; Gasparotti 2015).

Por otro lado, la recuperación de un recipiente cerámico en la E1 permitió indagar más profundamente en las actividades cotidianas realizadas en Corral Alto. Este hallazgo posibilitó realizar análisis de microrrestos vegetales para tratar de identificar qué estuvo en 
contacto con el recipiente. Se dedujo que esta vasija habría sido utilizada para el procesamiento de varios tipos de recursos vegetales como maíz, y posiblemente papa y porotos, entre otros (Gasparotti y Haros 2015). Conjuntamente con estas evidencias, la identificación de un endocarpo de chañar (Geoffroea decorticans) en la E1 (Escola et al. 2013) sugiere una variedad aún mayor de manejo de recursos vegetales locales y no locales por parte de los antiguos habitantes de Corral Alto.

Entonces, hasta el momento y partiendo de las evidencias recolectadas, es posible afirmar que en Corral Alto se realizaron diversas actividades domésticas a lo largo de un poco más de 200 años, como la cocción y almacenamiento de alimentos, el procesamiento y cocción de camélidos, la molienda de granos en los morteros fijos, la confección y uso de instrumentos líticos, y seguramente la crianza y cultivo de animales y plantas.

Pero, ¿qué papel jugaban los pobladores de Corral Alto en el contexto microrregional? ¿Qué rol tenía este sitio en el entramado social de este momento? Para esclarecer este panorama debemos tratar de aclarar el contexto socio-económico del Período Tardío en la microrregión de Antofagasta de la Sierra.

\section{El caso de estudio dentro del panorama regional}

En términos generales, el Período Tardío (ca. 1000-1450 DC) en el Noroeste Argentino ha sido caracterizado como un momento de desarrollos sociales regionales, conflictos, complejidad sociopolítica y desigualdad social. Inclusive se ha planteado la existencia de unidades políticas, del tipo jefaturas, que controlaban territorios específicos (Núñez Regueiro 1974; Tarragó 2000). Así, las primeras investigaciones describieron un proceso en el cual el crecimiento demográfico y el incremento de las prácticas agrícolas habrían desembocado en una paulatina modificación de la organización socio-política de las sociedades, provocando un mayor control político y una acentuada estratificación social a partir de ca. 700 años AP. Estas jefaturas se constituían en importantes centros de concentración de poder en los valles, mientras que las periferias, controladas por las élites dominantes, eran lugares de obtención de diversos recursos escasos en las cabeceras políticas.

Así, para la Puna meridional, se planteó un modelo de control vertical (sensu Murra 1975) entre el valle de Hualfín (1300 msnm) y las estepas puneñas (4000 msnm) (Raffino y Cigliano 1973; Tarragó 2000). Partiendo de esta concepción, en principio las investigaciones sobre el Tardío en Antofagasta de la Sierra se centraron en determinados sectores, específicamente en el fondo de cuenca del río Punilla (sensu Olivera 1992), y determinados sitios, como los grandes conglomerados habitacionales (p.ej. La Alumbrera) o extensas áreas 
de cultivo (p.ej. Bajo del Coypar). Esto reforzó la idea de la existencia de "enclaves" estratégicamente ubicados en zonas de concentración de recursos específicos (Raffino y Cigliano 1973; Vigliani 1999; Tarragó 2000; Tchilinguirian y Olivera 2000; Olivera y Vigliani 2000-2002; Olivera et al. 2003-2005; Olivera et al. 2008; Tchilinguirian y Olivera 2010).

Este panorama ha sido recientemente discutido en función de nuevos interrogantes que han traído aparejados la revisión de la cronología en el valle de Hualfín. Los fechados radiocarbónicos relacionados con las ocupaciones Belén parecen ser más tempranas en el área de Antofagasta de la Sierra que las de Hualfín, sin embargo esta situación merece un análisis más detallado, ya que las evidencias de ocupaciones más tempranas del período Tardío en el valle podrían haber sido enmascaradas por las reiteradas reocupaciones de los sitios (Wynveldt y Flores 2014). Este panorama hace que surjan nuevos interrogantes para futuras investigaciones.

Estos cuestionamientos acompañan el cambio de perspectiva que se está dando sobre este momento, lo que conjuntamente con las investigaciones realizadas en los sectores intermedios de la microrregión de Antofagasta de la Sierra, obligan a repensar el período Tardío. Para el caso particular de Antofagasta de la Sierra, el desarrollo de grupos corporativos orientados a actividades agrícolas extensivas y al control político-religioso en el fondo de cuenca no habría implicado necesariamente la desaparición de otros ejes económicos interfamiliares pastoriles en los sectores intermedios (Martel y Aschero 2007). En este contexto, la ocupación de Corral Alto podría corresponderse con la continuación de un modo de vida doméstico o cooperativo interfamiliar (Martel y Aschero 2007; Elías 2014; Grant y Escola 2015).

Esta propuesta podría asimilarse con una configuración sociopolítica segmentaria, en forma semejante a lo señalado por Nielsen (2006) para momentos tardíos en la quebrada de Humahuaca, sin desconocer, sin embargo, la posibilidad de la existencia de grupos de poder y el desarrollo de redes socio-económicas más centralizadas, coordinadas desde el fondo de cuenca, ocupada por los habitantes de La Alumbrera (Cohen 2011, 2014).

Cohen (2011) plantea que es posible que esta situación sea definida como un "archipiélago de poderes" de diferentes unidades familiares nucleares y extensas de antiguas tradiciones pastoriles en los sectores intermedios, que convivían con nuevas formas organizativas del fondo de cuenca. Es en este contexto de posible tensión y negociación de las élites de fondo de cuenca y las familias nucleares de sectores más alejados, donde se ubica Corral Alto. 
Entonces, ¿cómo pueden los estudios petrográficos echar luz sobre estas cuestiones? ¿Cómo a partir de un corte petrográfico podemos abrir el panorama hacia lo que sucede fuera del recipiente cerámico de donde proviene?

\section{La cerámica como objeto que ensambla y reúne relaciones}

Las cosas ensamblan en sí mismas toda una malla de conexiones y, cuando vemos los objetos como acabados, perdemos de vista la cantidad de materiales que están involucrados en ellos, aunque en realidad los materiales siguen allí y continúan mezclándose y reaccionando de la misma forma que siempre lo han hecho, reuniendo relaciones más allá de ellos (Ingold 2007). Al volver la mirada hacia el objeto se puede llegar a entender cómo los artefactos se convierten en memoria y marcadores sociales, cómo en un objeto se ven enredadas múltiples relaciones tanto con las personas, como con otras cosas (Hodder 2012).

Ya que son las cosas las que ensamblan, reúnen materiales, personas, relaciones, y es lo que perdura en el registro arqueológico, es a partir de los materiales involucrados en la elaboración de objetos cerámicos que se podría emprender un camino inverso. Es decir, desde las cosas hacia la red de relaciones en la cual se encuentran inmersas. En este sentido, las elecciones tecnológicas (sensu Lemonnier 1992, 1993) observables en el material cerámico son consecuencia de dicha red de relaciones, al igual que el "saber hacer" (o modo de hacer) tradicional y particular involucrado en la elaboración de cada objeto cerámico.

Al respecto, los estudios de petrografía cerámica contienen en sí mismos un doble valor, ya que permiten remitirse a las primeras elecciones tecnológicas tomadas por el alfarero, examinando, además de la composición de pastas, las materias primas utilizadas, lo que puede brindar una interpretación sobre procedencia de dichos recursos y posibilitar la reconstrucción de los modos de hacer.

Por lo tanto, este abordaje sobre petrografía y modos de hacer, nos puede llevar a la parte más irreductible del enredo (sensu Hodder 2012) y de alguna forma tratar de entender la red y las historias en las que los objetos están inmersos, historias que son el resultado de las relaciones entre objetos y personas, objetos y otros objetos, y personas con otras personas.

\section{Metodología y materiales}

Para comenzar a desandar este enredo material se realizó una caracterización de la composición y la cuantificación de los componentes de las pastas a través de secciones delgadas. Para esto se tuvieron en cuenta tres aspectos en cada muestra analizada: 
1. La descripción del fondo de pasta o matriz, (pseudolepidoblástica, microgranosa o criptofilitosa) (Curtois 1976; Cremonte y Pereyra Domingorena 2013).

2. La identificación mineralógica de las inclusiones mayores a $15 \mu \mathrm{m}$, y determinación de sus formas y tamaños.

3. La identificación y cuantificación de las cavidades o poros teniendo en cuenta sus formas y tamaños (Curtois 1976; Freestone 1991; Cremonte y Bugliani 2006-2009; Cremonte y Pereyra Domingorena 2013).

La cuantificación en cada caso se llevó a cabo mediante conteo de puntos (mínimo 300 por cada corte delgado observado a distancias constantes) con la utilización de un microscopio petrográfico de polarización (marca BIOTRAZA modelo XP-607 LPT). Estos datos fueron trabajados luego con un programa estadístico aplicando análisis de conglomerados (para el tratamiento de los datos y confección de gráficos se utilizó el programa PAST V. 3.02a (Hammer et al. 2001).

Esta metodología fue aplicada a una muestra de 37 fragmentos cerámicos. Estos provienen del numeroso conjunto recuperado de Corral Alto $(n=2171)$, que en primera instancia, fue sometido a un análisis a nivel macroscópico y sub macroscópico (con el uso de lupa binocular de bajos aumentos y a ojo desnudo). A través de esta primera etapa de análisis, se dividió el gran conjunto en 106 Grupos o Familias de fragmentos que posiblemente pertenecieron a un mismo recipiente. Esta fue la unidad mínima de análisis. A partir de ellos se reconstruyeron formas y en los casos en los que fue posible, se asignó un tipo estilístico. En cuanto a este punto se debe mencionar que muy pocas familias o grupos de fragmentos pudieron correlacionarse con algún tipo estilístico conocido (Gasparotti 2012, 2015).

Partiendo de las familias de fragmentos establecidas, se seleccionaron 37 muestras. Esta muestra pretendió ser representativa de la variabilidad observada en la primera etapa de análisis.

\section{Resultados}

En las 37 muestras se registraron distintas variables, entre las que se encuentran matriz, cavidades, cuarzo, plagioclasas, biotita, muscovita, clorita, cuarzo con extinción ondulante, gránulos de arcilla, piroxeno, anfíbol, olivino, hornblenda, microclino, minerales opacos, plagioclasa zonada, plagioclasa alterada a sericita, plagioclasa con venas de albita, pertita, rocas volcánicas no identificadas, vulcanita 1, vulcanita 23, basalto, dacita, riolita, andesita, ignimbrita, estructura con hábito esferulítico, vitroclastos, rocas graníticas, rocas 
metamórficas, arenisca, cuarcita, gneis, filita, esquisto, pizarra, rocas carbonáticas y tiesto molido.

Se debe mencionar que no todas estas variables fueron registradas en las 37 muestras analizadas, ya que la composición de las mismas no es homogénea. Una vez obtenidos los porcentajes de cada una de las variables se procedió a realizar el análisis estadístico de conglomerados. A través de este análisis, aplicando el método de pares agrupados (UPGMA) y mediante el índice de similitud euclidiano, se obtuvo un coeficiente cofenético de 0,7862 . A partir de este conglomerado, las muestras fueron separadas en nueve tipos petrográficos (Figura 3).

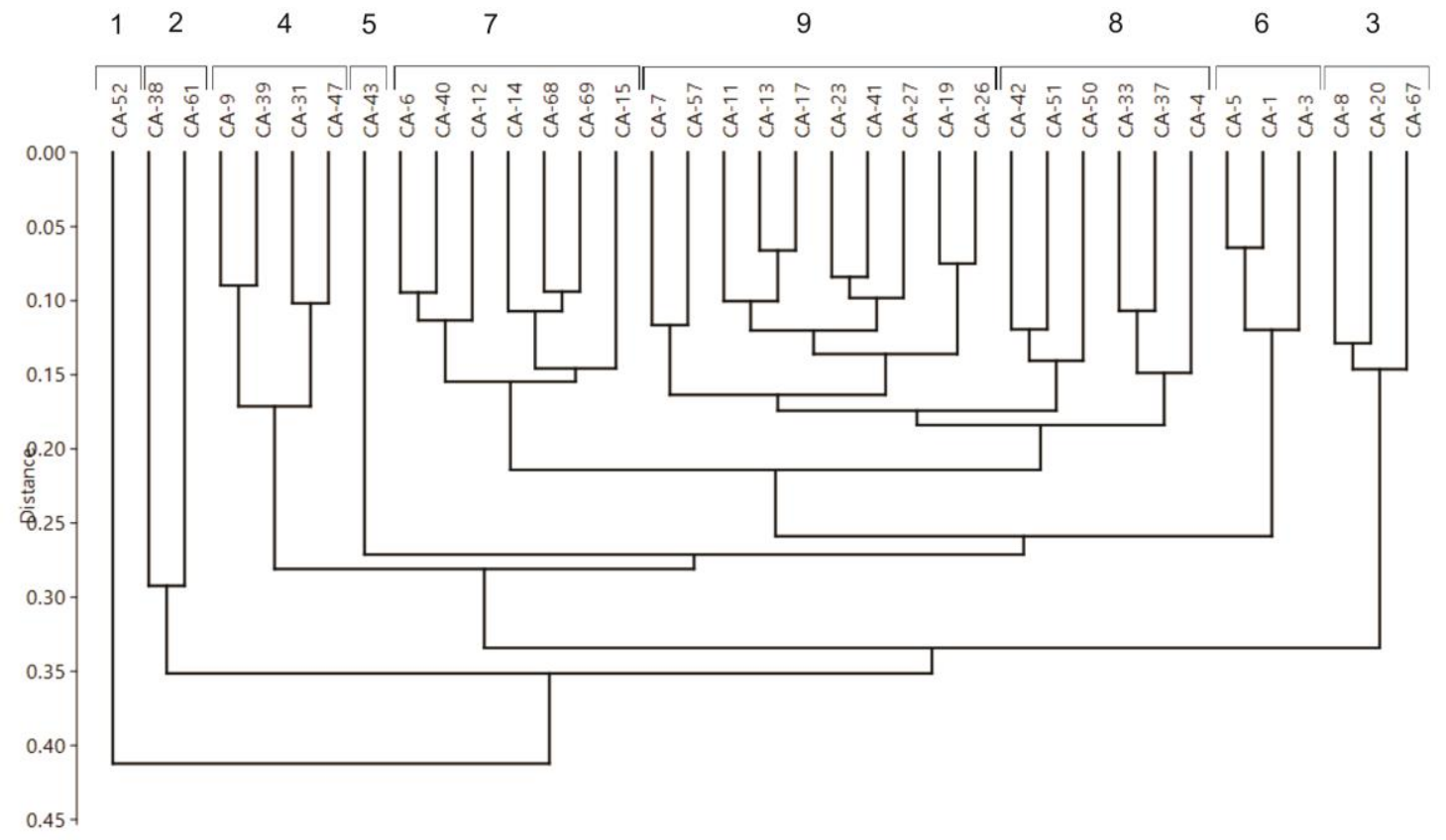

Figura 3. Diagrama de conglomerado conformado a partir de las 37 muestras analizadas.

Sobre la base de este análisis de conglomerado el conjunto puede ser divido en tres grandes grupos dependiendo de los porcentajes de inclusiones observados:

1- un grupo de "Pastas Gruesas" que es aquel que presenta predominio de inclusiones no plásticas, más de $54 \%$, sobre la matriz y cavidades (cinco tipos conformados por 10 muestras),

2- un grupo de las "Pastas Intermedias" que se configuró a partir de muestras con porcentajes de matriz entre $40 \%$ y $24 \%$, mientras que se contabilizaron inclusiones no plásticas entre $46 \%$ y $69 \%$ (dos tipos con 12 muestras), y 
3- Otro grupo que presenta mayor porcentaje de matriz (40\% o más) denominado "Pastas Finas" (dos tipos que engloban 14 muestras) (Figura 4).

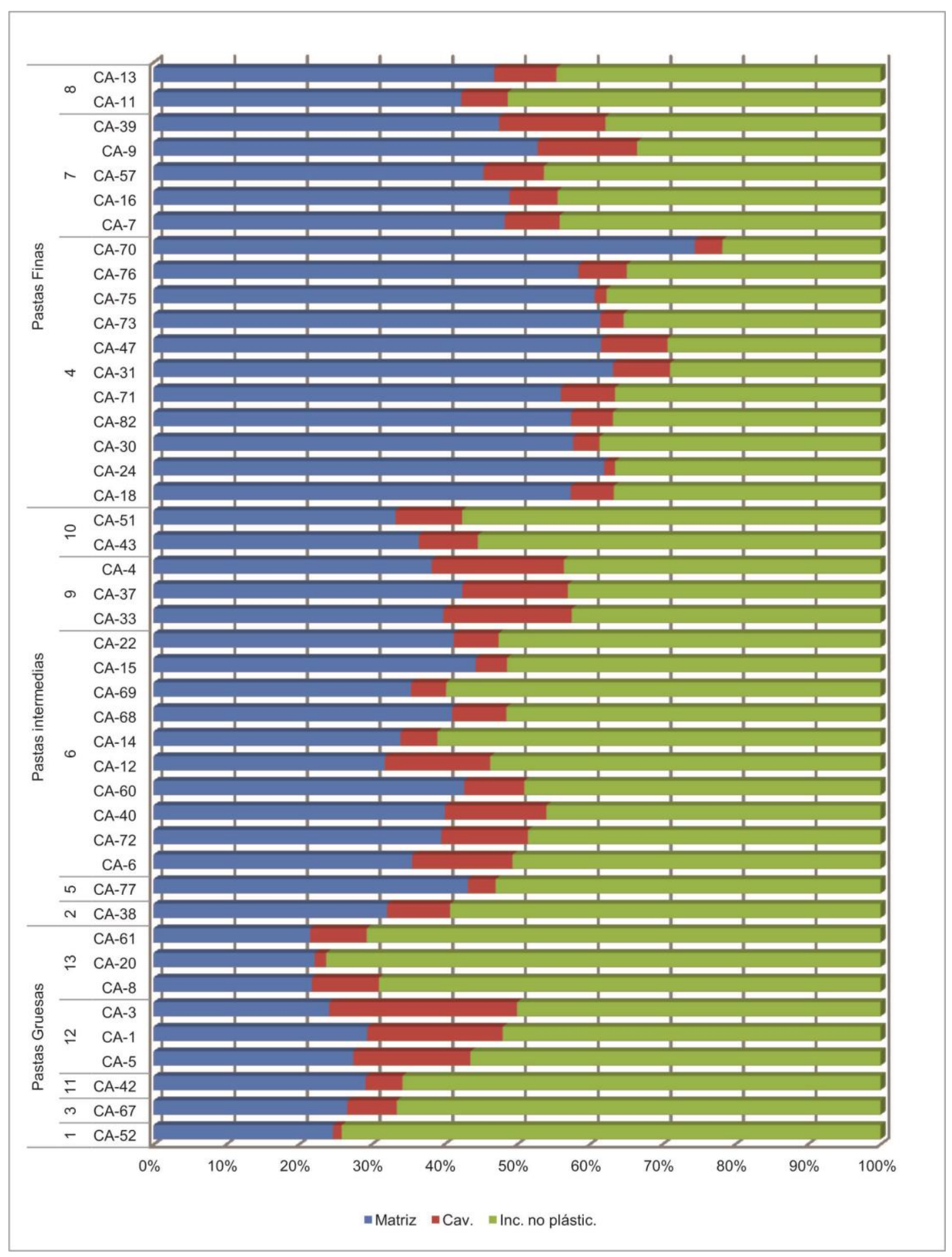

Figura 4. Divisiones entre los grupos de pastas y los porcentajes de matriz, cavidades e inclusiones no plásticas en cada una de las muestras. 


\begin{tabular}{|c|c|c|}
\hline $\begin{array}{l}\text { Grupo de } \\
\text { Pasta }\end{array}$ & Corte & Matriz o fondo de pasta \\
\hline PG & $\mathrm{CA}-1$ & Microgranosa \\
\hline PG & CA-3 & Microaranosa \\
\hline$\overline{P G}$ & CA-5 & Criptofilitosa y algo microgranosa \\
\hline PG & $\mathrm{CA}-8$ & $\begin{array}{c}\text { Microgranosa y algo } \\
\text { pseudolepidoblástica }\end{array}$ \\
\hline PG & CA-20 & $\begin{array}{l}\text { Microgranosa y algo } \\
\text { pseudolepidoblástca }\end{array}$ \\
\hline PG & CA-38 & $\begin{array}{l}\text { Pseudolepidoblástica y algo } \\
\text { microgranosa }\end{array}$ \\
\hline PG & CA-43 & Pseudolepidoblástica \\
\hline PG & CA-52 & Microgranosa \\
\hline PG & CA-61 & Pseudolepidoblástica \\
\hline PG & CA-67 & $\begin{array}{l}\text { Pseudolepidoblástica y algo } \\
\text { microgranosa }\end{array}$ \\
\hline PIN & $\mathrm{CA}-4$ & Microaranosa \\
\hline PIN & CA-6 & Microgranosa \\
\hline PIN & CA-12 & $\begin{array}{l}\text { Pseudolepidoblástica y algo } \\
\text { microgranosa }\end{array}$ \\
\hline PIN & CA-14 & $\begin{array}{c}\text { Pseudolepidoblástica y algo } \\
\text { microaranosa }\end{array}$ \\
\hline PIN & CA-15 & $\begin{array}{c}\text { Microgranosa y algo } \\
\text { pseudolepidoblástica }\end{array}$ \\
\hline PIN & CA-33 & $\begin{array}{c}\text { Pseudolepidoblástica y algo } \\
\text { microaranosa }\end{array}$ \\
\hline PIN & CA-37 & $\begin{array}{l}\text { Pseudolepidoblástica y algo } \\
\text { microaranosa }\end{array}$ \\
\hline PIN & $\mathrm{CA}-40$ & Microgranosa \\
\hline PIN & $\mathrm{CA}-42$ & $\begin{array}{l}\text { Microgranosa y algo } \\
\text { pseudolepidoblástca }\end{array}$ \\
\hline PIN & CA-50 & $\begin{array}{c}\text { Pseudolepidoblástica y algo } \\
\text { microgranosa }\end{array}$ \\
\hline PIN & CA-51 & $\begin{array}{c}\text { Pseudolepidoblástica y algo } \\
\text { microaranosa }\end{array}$ \\
\hline PIN & CA-68 & Criptofilitosa \\
\hline PF & CA-7 & Microgranosa \\
\hline PF & CA-9 & Pseudolepidoblástica \\
\hline PF & $\mathrm{CA}-11$ & $\begin{array}{l}\text { Pseudolepidoblástica y algo } \\
\text { microgranosa }\end{array}$ \\
\hline PF & $\mathrm{CA}-13$ & $\begin{array}{l}\text { Microgranosa y algo } \\
\text { pseudolepidoblástca }\end{array}$ \\
\hline PF & CA-17 & Microgranosa \\
\hline
\end{tabular}

Tabla 3. Muestras de secciones delgadas y sus respectivas matrices o fondos de pasta observados. Además, se identificó en qué grupo de pasta se encuentra cada muestra: PG: pasta gruesa, PIN: pasta intermedia y PF: pasta fina.

Pastas Gruesas:

Este grupo presenta como denominador común que todos los casos muestran mayor porcentaje de inclusiones no plásticas y cavidades que de matriz, siempre superando el 54\% hasta un 78\%. Sin embargo, existe una importante variabilidad dentro de este grupo, siendo las pastas gruesas las que más tipos engloban (5). 
En cuanto a composición, en las pastas de este grupo se observa que la mayoría de las muestras tienen presencia de rocas volcánicas. Los porcentajes de matriz nunca superan el $32 \%$, y las cavidades el $10 \%$.
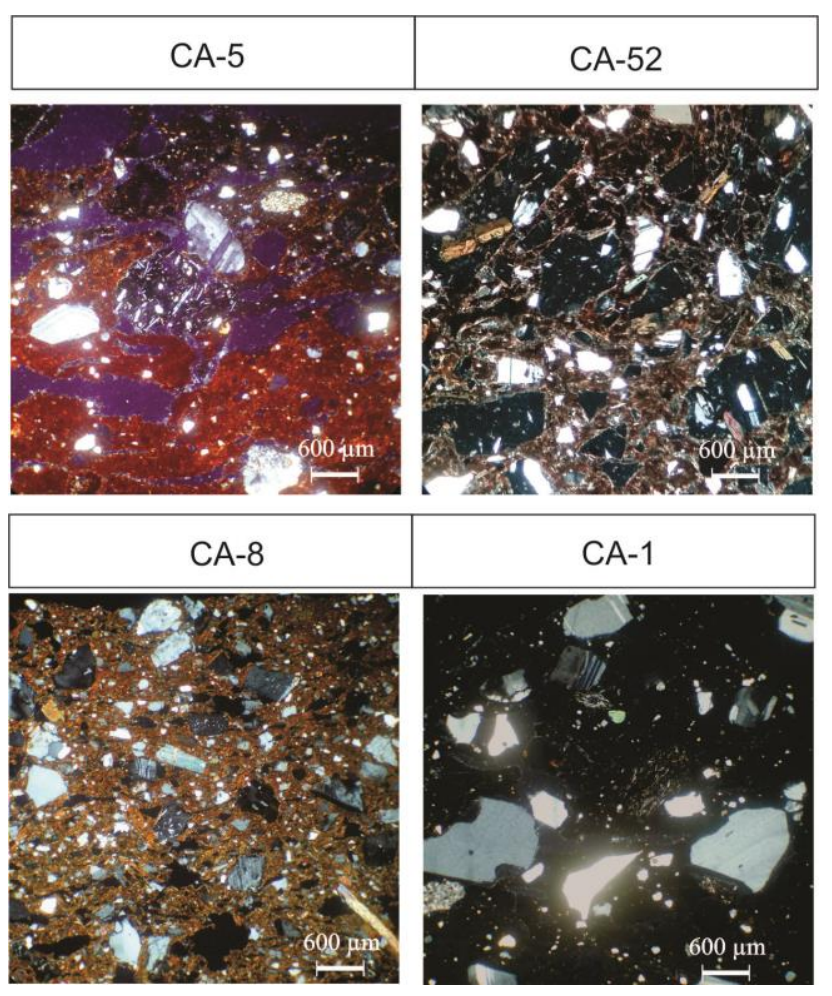

Figura 5. Fotomicrografías de muestras pertenecientes al grupo de Pastas Gruesas. Tomadas en luz polarizada.

Por otro lado, las medidas de las inclusiones no plásticas de este conjunto de pastas son bastante heterogéneas y van desde los limos gruesos hasta guijarros (según la escala granulométrica de Udden-Wentworth). En cuanto a las matrices predominan aquellas microgranosas, aunque hay muchas variaciones y combinaciones.

Dentro de este grupo se observa variabilidad en cuanto a las formas identificadas, aunque predominan las ollas abiertas (3) y cerradas (2), seguidas por las formas indeterminadas (4) y un puco (1).

Este grupo incluye los Tipos 1, 2, 3, 5 y 6. El tipo 1 es el que presenta la mayor diferencia. Se compone por una sola muestra que se destaca por presentar altos porcentajes de ignimbritas (31,82\%). Mientras tanto, el Tipo 2 (dos muestras) se caracteriza por registrar importantes porcentajes de cuarzo y plagioclasas. Por otro lado, el Tipo 3 (tres muestras) se 
identifica por los altos contenidos de cuarzo, plagioclasa, biotita, microclino y minerales opacos, en sentido decreciente.

El Tipo 5, compuesto por una sola muestra, presenta altos porcentajes de cuarzo, plagioclasa y biotita. También se identificaron cristaloclastos de plagioclasa sericitizada.

Finalmente, el Tipo 6 se conforma por 3 muestras, se caracteriza por contener los porcentajes más elevados de $\mathrm{Vc}^{3}{ }^{3}$ y andesitas. Este tipo engloba la cerámica $\mathrm{N} / \mathrm{R}$ posiblemente relacionada con Belén.

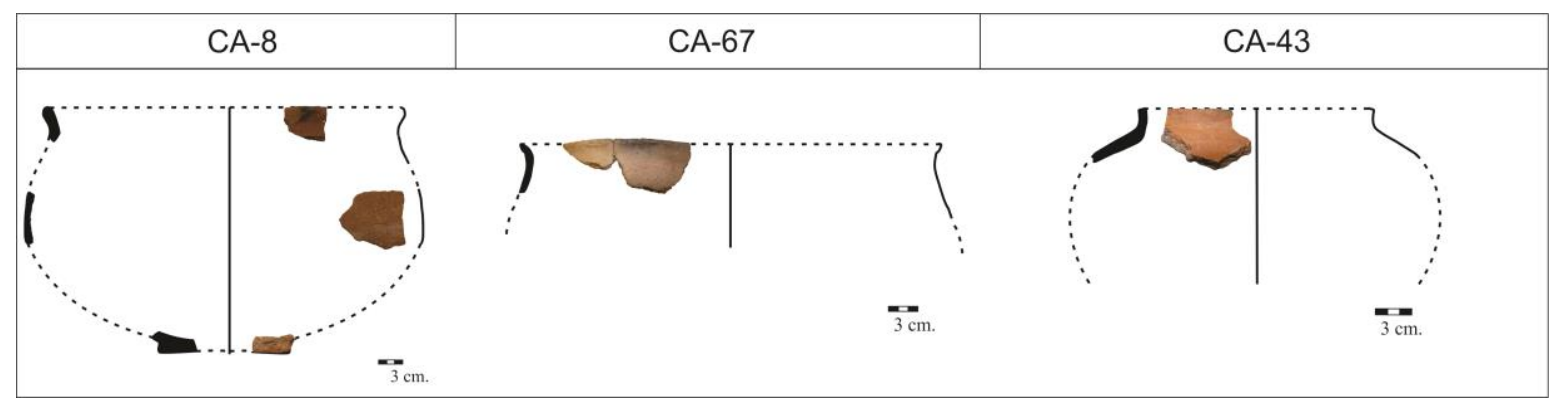

Figura 6. Reconstrucción de algunas formas pertenecientes al grupo de Pastas Gruesas.

Pastas intermedias

Este grupo se caracteriza por presentar: a) porcentajes de matriz que van desde el $25 \%$ al $40 \%$, b) inclusiones no plásticas desde $53 \%$ a $69 \%$, y c) cavidades entre un $5 \%$ a un $20 \%$. Como se puede observar es un grupo más heterogéneo. Incluye dos tipos de pastas los cuales tiene la mayor cantidad de rocas graníticas de toda la muestra.

En la observación de las medidas de las inclusiones no plásticas se detectó una variabilidad que va desde limos medios a gránulos (según la escala granulométrica de Udden-Wentworth). En comparación con los otros dos grupos, éste es el que presenta inclusiones de menor tamaño general.

Respecto a los tipos de matriz o fondos de pasta predominan las estructuras microgranosas y pseudolepidoblásticas algo microgranosas, aunque hay variabilidad.

Respecto a las formas identificadas dentro de este grupo predominan las ollas de perfil abierto (6), seguidas por las indeterminadas (3), las ollas de perfil cerrado (2) y un puco (1). Este grupo involucra los Tipos 7 y 8. El Tipo de pasta 7, compuesto por seis muestras, muestra presencia de cuarzo, por encima del 18\%, así también como plagioclasas, biotitas y 

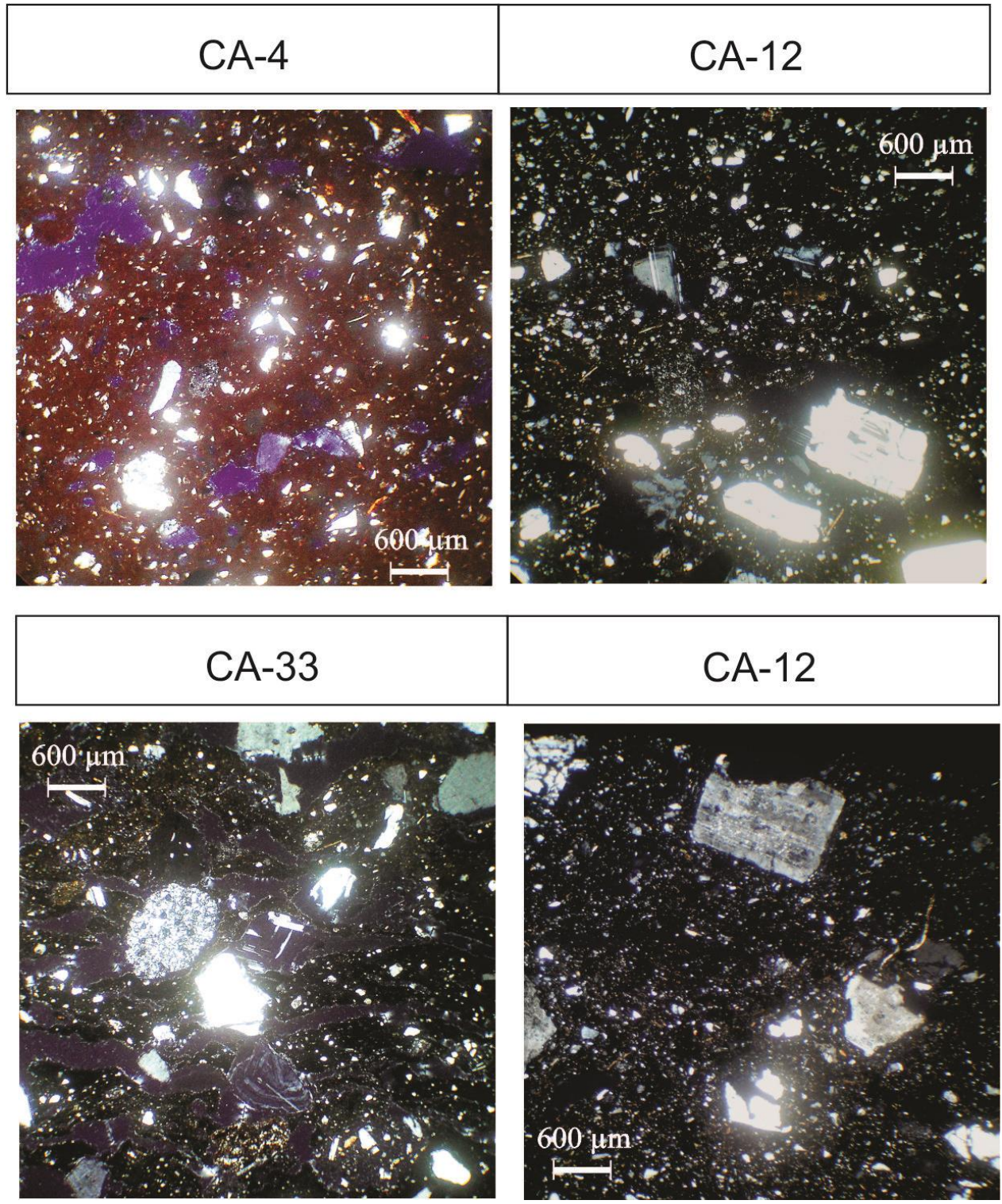

Figura 7. Fotomicrografías de muestras pertenecientes al grupo de Pastas Intermedias. Luz polarizada.

muscovitas. Además, aunque en pocas cantidades, todas las muestras presentan diversas rocas volcánicas. El Tipo 8, conformado por seis muestras, se distingue por la alta proporción de cuarzo, por encima del $35 \%$. Todas las muestras contienen minerales opacos y vulcanita 2 . 
Pastas finas

Finalmente, los tipos que conforman el grupo de pastas finas resultan más homogéneos en general. Los porcentajes de matriz nunca son inferiores al 39\% y pueden llegar hasta el $60 \%$.

En este grupo las dimensiones observadas para las inclusiones no plásticas van desde limos gruesos a gránulos, ubicándose en medio de los dos grupos anteriores en cuanto a tamaños (según la escala granulométrica de Udden-Wentworth). En cuanto a los fondos de pasta, en general, predominan aquellos pseudolepidoblásticosm seguidos por los microgranosos y algo pseudolepidoblásticos.

Para este último grupo se identificaron algunas formas, entre las que se reconocieron predominantemente ollas de perfil abierto (5), seguidas de las formas indeterminadas (3), ollas globulares (2), ollas de perfil cerrado (2) y pucos (2).

Finalmente, este grupo está conformado por los Tipos 4 y 9. El Tipo 4 se compone por 4 muestras, y se caracteriza por presentar los porcentajes más elevado de matriz de toda la muestra (por encima de 43\%). Asimismo, en todas las muestras de este Tipo se identificaron inclusiones de cuarzo, plagioclasa y biotitas, además de diferentes rocas volcánicas y ciertas rocas graníticas. Algunos de los ejemplares de este tipo de pasta presentan tiesto molido, aunque en escasa proporciones. Respecto a los fondos de pasta, en general predominan los pseudolepidoblásicos, seguidos de los microgranosos y algo pseudolepidoblásticos.

Finalmente, el Tipo 9, conformado por 10 muestras (el más numeroso del conjunto), presenta un relativo equilibrio entre los porcentajes de matriz (entre 39\% a 44\%) e inclusiones no plásticas (entre $46 \%$ a 54\%). Todas las muestras presentan cuarzo y plagioclasa, minerales opacos y rocas volcánicas.

En síntesis, respecto del total de muestras analizadas $(n=37)$, se puede decir que se trata de un conjunto bastante heterogéneo, que pudo ser divido en tres grandes grupos, en las Pastas Gruesas predominan las matrices microgranosas, en las Pastas Intermedias predominan las pseudolepidoblásticas y algo microgranosas, mientras que en las Pastas Finas resaltan las texturas pseudolepidoblásticas. Tal vez esta característica sugiera la utilización de distintas fuentes de arcillas. 


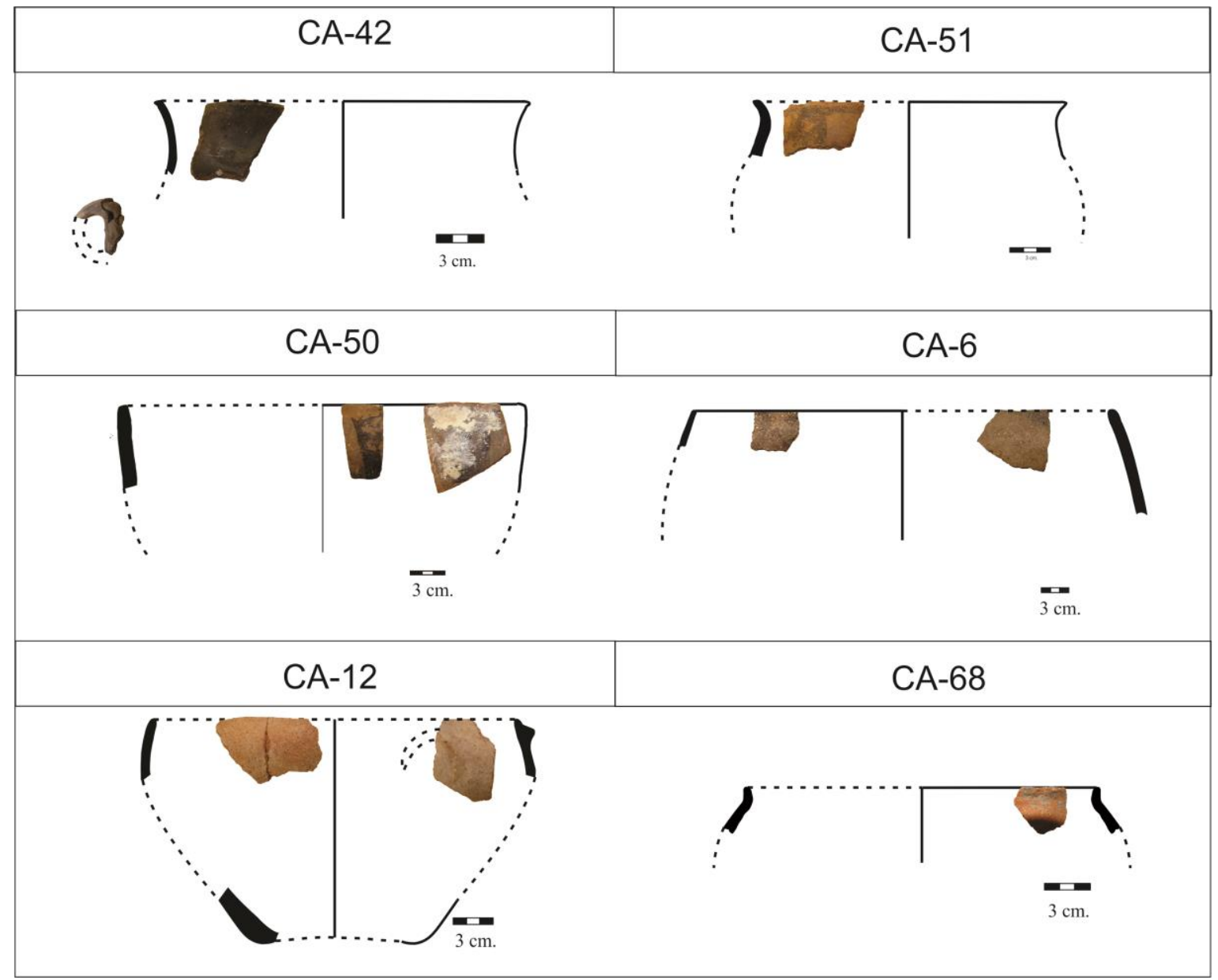

Figura 8. Reconstrucción de algunas formas pertenecientes al grupo de Pastas Intermedias.

\section{Elecciones tecnológicas en la petrografía de Corral Alto}

Los tres grupos definidos (pastas gruesas, intermedias y finas) presentan pocas diferencias en cuanto a las inclusiones no plásticas identificadas, con presencia en todos ellos de cuarzo, plagioclasas, biotita, muscovita, rocas volcánicas y rocas graníticas. En términos generales, no se observaron tendencias claras de estándares de pastas, sino variabilidad dentro del conjunto. Sin embargo, hay algunos Tipos que se diferencian claramente, como el Tipo 1, con altos porcentajes de ignimbritas, y el Tipo 5 que presenta porcentajes llamativos de plagioclasas sericitizadas. 
Por otro lado, es interesante notar que el grupo de las Pastas Gruesas se presenta mucho más heterogéneo en su interior en cuanto a su composición a pesar de agrupar menor cantidad de muestras. Por el contrario, el grupo de las Pastas Intermedias y las Pastas Finas, a pesar de aglomerar mayor cantidad de muestras, éstas son más homogéneas entre sí, dando como resultado una menor cantidad de tipos petrográficos.

Por lo tanto, se puede afirmar que las elecciones tecnológicas referidas a las Pastas Gruesas son más flexibles y heterogéneas que en los otros dos grupos, es decir que pudieron existir varios modos de hacer coexistiendo en el mismo momento y lugar.

Por último, sobresale la muestra CA-38, aglomerada en el Tipo 2. Esta muestra presenta cantidades llamativas de tiesto molido (12,76\%), lo que claramente refleja una elección tecnológica diferente, ya que no podría considerarse ocasional la inclusión de este tipo de antiplástico.

Ahora bien, ¿cómo estas elecciones tecnológicas se relacionan con la geología local y la posible proveniencia de las materias primas? La geología local está conformada prácticamente en su totalidad por sedimentitas y vulcanitas cenozoicas. La zona cercana a Corral Alto se caracteriza por presentar vulcanitas, las que componen afloramientos que cubren casi el 70\% de la superficie estudiada (Aceñolaza et al. 1976; González 1992). Esta característica se ve ratificada por el estudio petrográfico que he realizado a distintas arenas procedentes de la quebrada del río Miriguaca. Se observa la predominancia de litoclastos volcánicos como andesitas, vulcanitas e ignimbritas, además de cuarzos y plagioclasas (Figura 11).

Podemos proponer una notable vinculación entre la mayoría de los tipos petrográficos y la geología regional, ya que las inclusiones de rocas volcánicas son una constante dentro de las muestras de arenas estudiadas. Para el caso de la quebrada del río Las Pitas, Puente (2015) también ha realizado estudios sobre las arenas locales, identificado el predominio de componentes volcánicos. Estos componentes muestran relación con ciertos conjuntos de piezas analizadas.

Por otro lado, se torna visible la ausencia de una correspondencia entre tipos petrográficos, formas identificadas y los estilos decorativos. Entonces, hasta el momento, no se han observado tipos de pastas específicos para determinadas formas o estilos particulares, sino más bien parece haber existido una cierta flexibilidad en cuanto a las elecciones tecnológicas tomadas por los alfareros. 

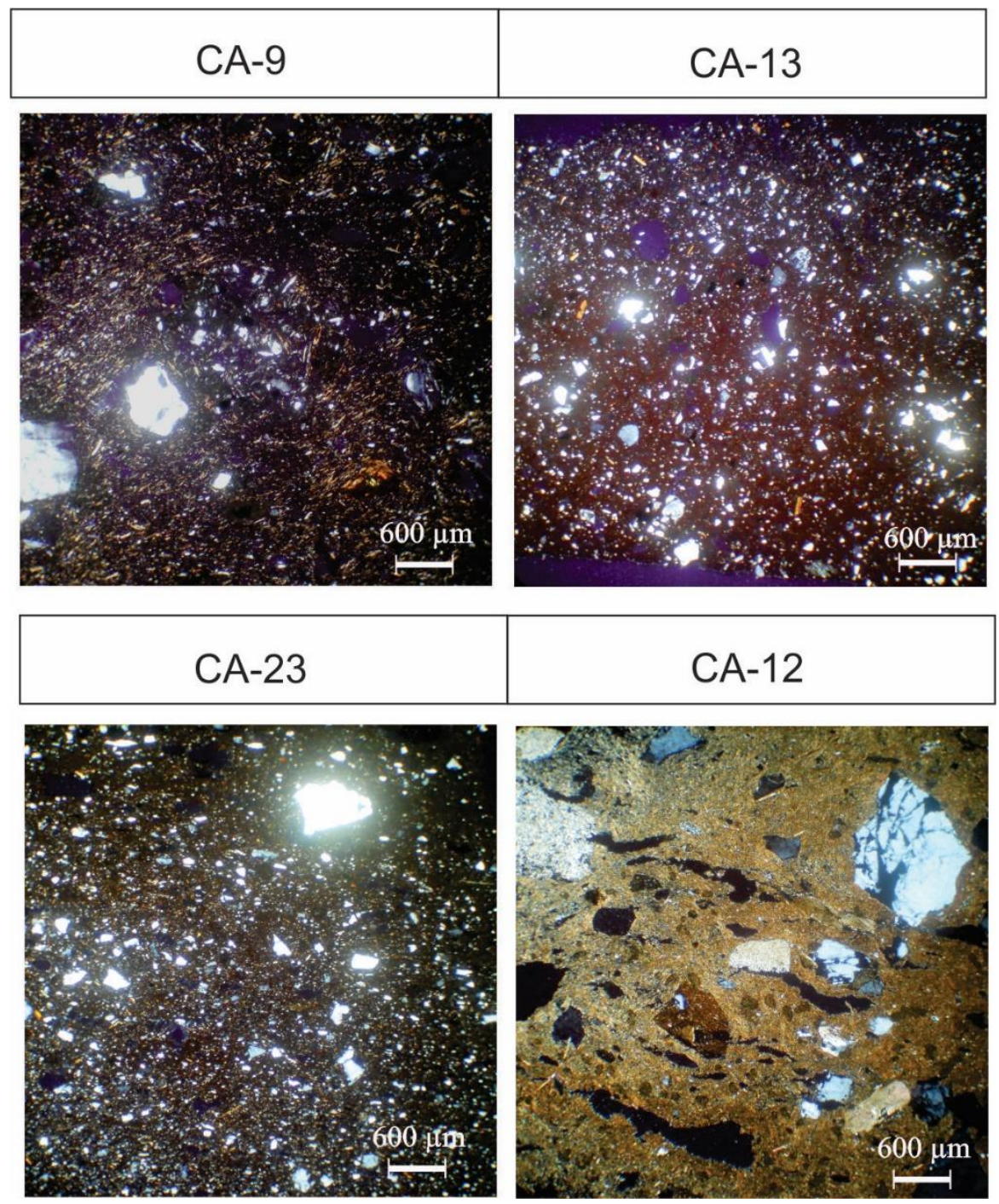

Figura 9. Fotomicrografías de muestras pertenecientes al grupo de Pastas Finas. Luz polarizada.

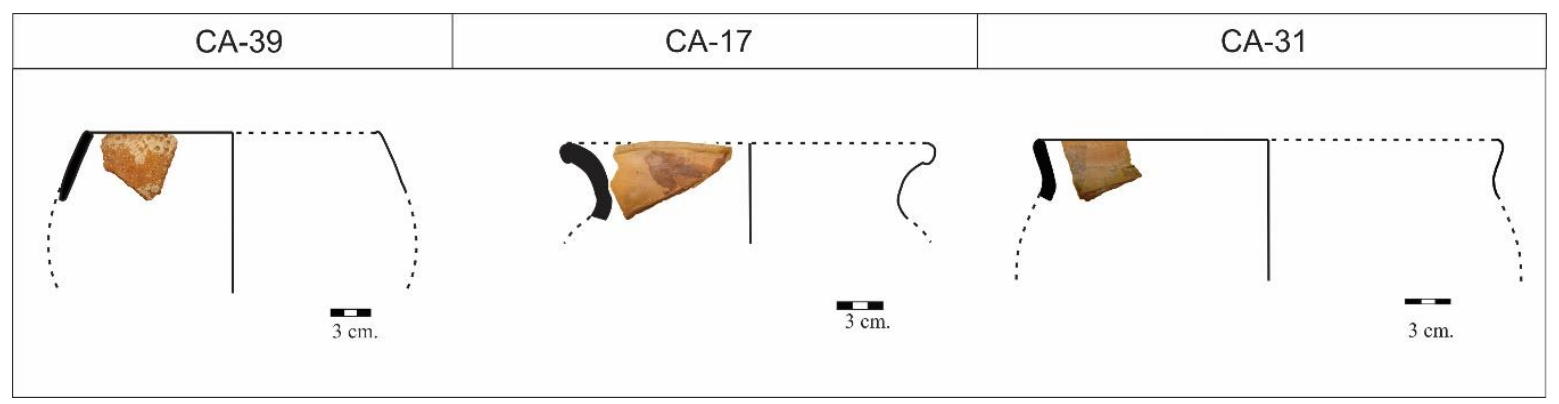

Figura 10. Reconstrucción de algunas formas pertenecientes al grupo de Pastas Finas. 

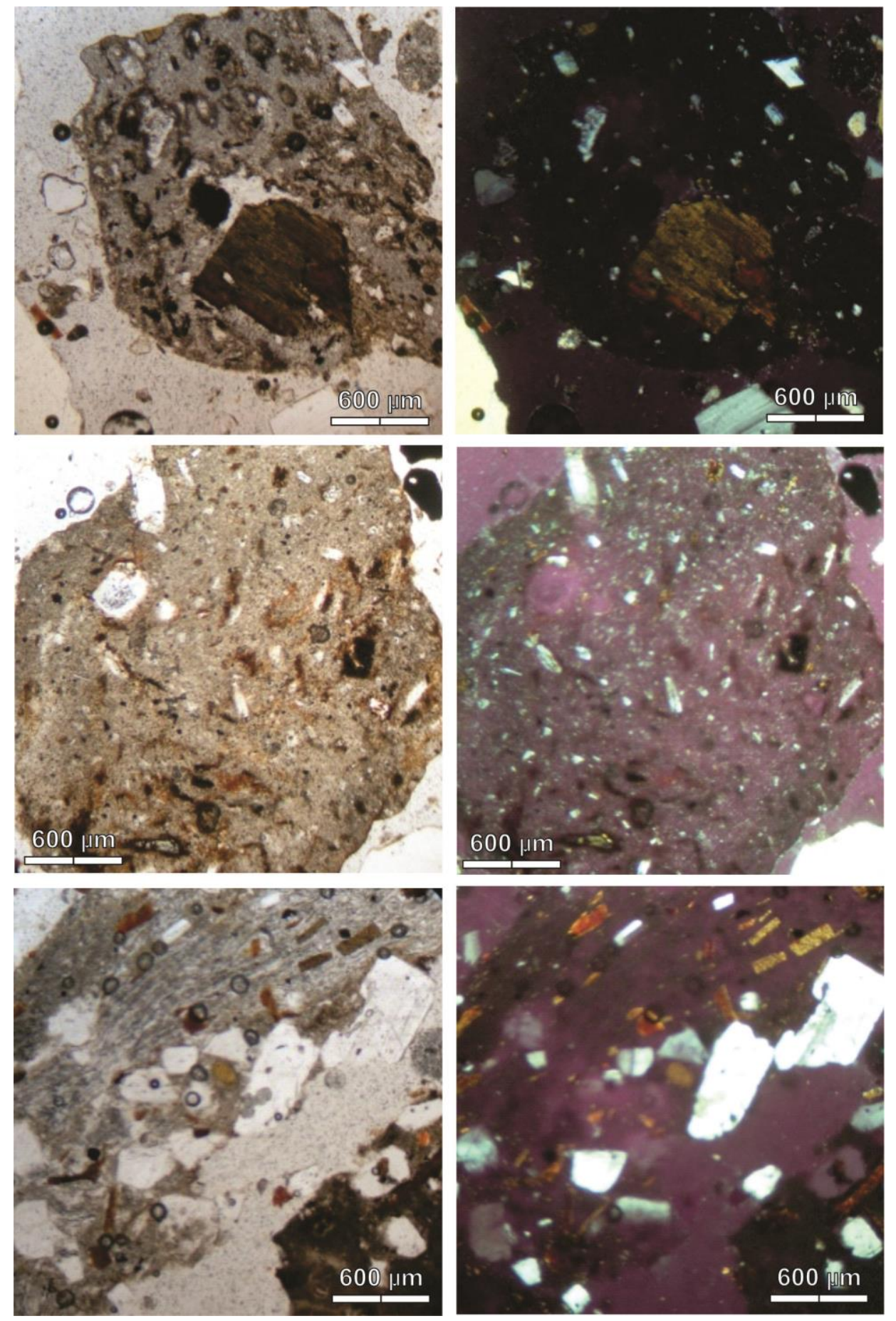

Figura 11. Arenas tomadas en las cercanías de Corral Alto.

Fotomicrografías en microscopio de polarización (Izq. Luz paralela, Der. Luz polarizada). 


\section{Reflexiones a modo de conclusión}

Es difícil plantear una conclusión en este trabajo cuando aún resta tanto por investigar, en cambio, intentaré ensayar algunas reflexiones que la investigación hasta aquí desarrollada me ha permitido alcanzar.

Como primer punto creo necesario destacar que el conjunto cerámico de Corral Alto puedo ser definido como de carácter doméstico, resultado de una producción de baja escala orientada a resolver necesidades básicas de un grupo reducido de personas. Entre las actividades inferidas a partir de este equipo cerámico se encontraría el almacenaje de alimentos y líquidos, la cocción y el servicio de los mismos. Se podría pensar que el almacenaje de productos secos, como granos, habría sido una de las actividades más comunes, debido a la alta cantidad de vasijas aptas para este propósito (de las 39 vasijas reconstruidas, posiblemente 26 podrían corresponderse con actividades de almacenaje, tratándose de ollas con y sin cuello o tinajas). Esta situación podría asociarse con el aumento de las actividades de producción agrícola que se habrían generado durante el Tardío a nivel regional. Esta nueva orientación en la subsistencia también se ve reflejada en Corral Alto a través de la gran cantidad de morteros fijos registrados, en los campos de cultivo relevados en las cercanías asociados cronológicamente a este momento. Asimismo, el almacenaje de agua debió haber sido un punto crítico, ya que el acceso hacia la fuente más cercana se ve restringida por la ubicación de este asentamiento.

En segundo lugar, específicamente en lo que respecta a los análisis petrográficos, se observó cierta variabilidad en las materias primas utilizadas para la elaboración de los recipientes cerámicos, principalmente dentro del grupo de Pastas Gruesas. En este sentido, la heterogeneidad detectada en la composición de las pastas hace referencia a que, a pesar de dominar los componentes de origen volcánico, no hay estándares en la selección de materas primas, ya que las densidades en los componentes varían notablemente, lo cual podría responder al uso de fuentes de materias primas similares pero con distinto tipo de selección en sus componentes. De esta manera, la ausencia de correspondencia entre tipos de pasta y formas o estilos decorativos permite afirmar la existencia de elecciones tecnológicas flexibles, no pautadas ni estandarizadas.

Entonces, vale la pena preguntarse si el uso de materias primas diferentes se corresponde con la elección consciente de terminadas fuentes o, si bien, a la utilización de materias primas macroscópicamente similares, con excepción de algunos casos, como el tiesto molido o la ignimbrita. En términos generales, las materias primas parecen tener un origen local, y la variabilidad observada podría corresponder a la utilización varias fuentes de materias 
primas ubicadas a lo largo de la quebrada, o bien una misma fuente en la que las arenas se encuentren poco seleccionadas.

En este sentido, la utilización de este tipo de materias primas para la elaboración de la mayoría de las piezas hace referencia a alfareros que aprovecharon al máximo los recursos locales, entendiendo que éstos funcionarían de igual manera para la confección de distintos tipos de formas. También podría inferirse que estos alfareros estaban más arraigados a su entorno local, lo cual hablaría de poblaciones sedentarias que desarrollan una economía diversificada, con un mayor énfasis en lo agrícola, lo cual implicaría tener una menor disponibilidad de tiempo para abordar todo el rango de actividades relacionadas con la subsistencia. En este contexto, sería lógico que recurrieran a la utilización de materias primas inmediatamente disponibles para la elaboración de la cerámica.

En definitiva, se plantea la hipótesis de una manufactura local de gran parte del conjunto, como al parecer también sucede en la quebrada de Las Pitas, según los trabajos de Puente (2015). Este dato no debe llevar a descartar que puedan haber existido modos de hacer compartidos con otros alfareros, probablemente de los valles mesotermales o del fondo de la cuenca de Antofagasta de la Sierra. Estos modos de hacer diferentes se verían reflejados, por ejemplo, en la práctica de agregar tiesto molido, práctica que se ha identificado en la alfarería estudiada en los sitios de La Alumbrera y Bajo del Coypar II (Pérez 2013) o en el Valle del Bolsón (Puente 2015).

Resulta interesante destacar que la práctica de agregar tiesto molido a las pastas cerámicas, que hasta ahora tiene una escasa representación en Corral Alto, ha sido observada, e incluso en algunos casos es un elemento predominante, en el fondo de cuenca, específicamente en los sitios de La Alumbrera y Bajo del Coypar II, los cuales se emplazan a unos $17 \mathrm{~km}$ aproximadamente de distancia de Corral Alto (Pérez 2013). Esta evidencia sugiere que los alfareros habrían tomado elecciones tecnológicas diferentes, que podrían hacer referencia a distintos modos de hacer entre aquellos alfareros de fondo de cuenca y lo que habitaron los sectores intermedios de las quebradas subsidiarias (Pérez y Gasparotti 2016). Como plantea Puente (2010) este tipo de elecciones tecnológicas podrían estar relacionadas con modos de hacer característicos de los valles. Esta práctica ha sido registrada en el Valle del Bolsón (Puente 2012), en el Valle de Santa María (Palamarzuk 2008), en Tafí del Valle (Páez 2010) e incluso en Andalgalá (Cremonte 1991).

Estas prácticas de producción alfarera, que pudieron ser identificadas a través de la petrográfica cerámica, conducen a la compleja red de relaciones de la cual son resultado. Una red de relaciones entre las personas, los objetos y el paisaje de la Quebrada de Miriguaca que dieron como resultado estas vasijas confeccionadas de esta manera particular 
y no de otra. En este sentido, el conjunto cerámico de Corral Alto poco tiene que ver con un estándar de producción cerámica, como tampoco con los datos petrográficos provenientes del fondo de cuenca. A pesar que para los casos de La Alumbrera y Bajo del Coypar II se ha propuesto una asociación de las muestras analizadas petrográficamente con la geología regional, la diferencia observada con el conjunto de Corra Alto reside en que, por ejemplo, un porcentaje de la muestra asociado estilísticamente a Santa María, se vincularían geológicamente con el área de los valle mesotermales, asociando las características de esta pasta a una manifestación identitaria (Pérez 2013). Además, como ya se mencionó anteriormente, las muestras con inclusiones de tiesto molido tampoco son comunes en Corral Alto. Estas, en términos generales, podrían ser las particularidades que caracterizaron las elecciones tecnológicas en los sectores intermedios.

A partir de estas evidencias, podría pensarse, como ya fue planteado por Martel y Aschero (2007), sería posible que diferentes grupos inter-familiares asentados en las quebradas de los sectores intermedios se encontraran resistiendo o negociando constantemente el nuevo orden socio-económico con los grupos asentados en el fondo de cuenca en el gran conglomerado de La Alumbrera. De este modo, conservarían un modo de vida orientado al sustento de grupos familiares, en las que prácticas de mayor profundidad temporal se habrían preservado en cierta medida, como también se ve reflejado en los resultadas obtenidos desde otras materialidades, como los alcanzados por Grant (2014) y Elías (2010) sobre las evidencias faunísticas y líticas. Esta situación también se ve evidenciada en los propios modos de hacer cerámica, que muestran elecciones particulares y diferentes con relación a aquellas de fondo de cuenca.

Entonces, a partir de los estudios realizados sobre la alfarería de Corral Alto fue posible indagar en las cualidades de los materiales, que ensamblan en sí mismos toda una red de conexiones, tanto con otros objetos, personas y lugares, comenzar a investigar en las redes que se extienden más allá del sitio, y que atraviesan amplios espacios y paisajes, que hablan de relaciones y de distancias, que cuentan historias de objetos, de alfareros, de lugares y de experiencias. Redes que empiezan a vislumbrarse y que tal vez conducen a comprender una parte del contexto social del Período Tardío en la Quebrada del río Miriguaca.

Agradecimientos: en primer lugar quisiera agradecer a Patricia Escola por su con constante ayuda en todas las etapas de este trabajo. También a los geólogos Gustavo Toselli y Matías Rasjido quienes colaboraron con la identificación petrográfica. Los comentarios de los evaluadores fueron muy valiosos para este trabajo, agradezco su esfuerzo y generosidad. A todos aquellos que participaron en los trabajos de campo y permitieron que hoy pueda contar con estos materiales, especialmente a Natalia Sentinelli y Lorena Grana. Finalmente 
quiero agradecer a los organizadores del simposio que convocaron para la realización de este dossier.

\section{Notas}

1- El concepto fue propuesto por Aschero (1988:23), quien la define como "una serie de microambientes o zonas con recursos topográficos y vegetales diferenciados que se presentan en una cierta continuidad espacial y que representan una muestra adecuada del potencial de recursos que ofrece la región geográfica en estudio". Cabe destacar que dicha definición de micro-región puede hacerse extensiva a todo tipo de recursos naturales (faunísticos, minerales, etc.).

2- Olivera (1992) definió, dentro de la micro-región, tres sectores microambientales, que se diferencian en cuanto a sus características ecológicas y topográficas, así como también en lo que refiere a la oferta de recursos faunísticos, vegetales y minerales: A) Fondo de cuenca, entre los 3400 y 3550 msnm, con vegetación de vega, tolar y campo. Este sector brinda las mejores posibilidades para la agricultura, ya que su topografía abierta y la disponibilidad de agua permanente permiten la existencia de suelos aptos. Por otro lado, los recursos animales registrados son Lama glama, Rhea sp., Ctenomys sp. y aves acuáticas. B) Sectores intermedios, entre los 3550 y 3800 msnm. Predominan las vegas, el tolar y el campo. Los sectores de quebradas con vegas son los más aptos para la producción agrícola en baja escala y pastoril. Los recursos animales registrados son Lama glama, Rhea sp., Lagidium sp., Ctenomys sp. y aves. Cabe aclarar que los cursos inferiores de los ríos Las Pitas y Miriguaca son considerados parte de éstos, a pesar de registrar una altitud de $3400 \mathrm{msnm}$ (Olivera 1992; Escola et al. 2013). C) Quebradas de altura, entre los 3800 y 4600 msnm. Se conforman en los cursos medios y superiores de los ríos tributarios. Son, en general, quebradas relativamente estrechas y protegidas, donde corren cursos de agua permanentes. Aquí se registra vegetación de vega y pajonal, y recursos animales como Lama glama, Vicugna vicugna, Lagidium sp., otros roedores y aves. Son lugares principalmente aptos para el pastoreo.

3- En al análisis petrográfico de esta muestra se identificaron dos tipos de vulcanitas, las cuales fueron denominadas Vc1 y Vc2. La primera se compone de pequeñas agujas de plagioclasas envueltas en una matriz vítrea. Mientras tanto la Vc2 se caracteriza por presentar fenocristales más desarrollados de plagioclasas, entre otros, también dentro de una matriz más o menos vítrea. 


\section{Bibliografía citada}

Aceñolaza, F.; Toselli, G. y O. González

1976 Geología de la región comprendida entre el Salar del Hombre Muerto y Antofagasta de la Sierra, Provincia de Catamarca. Revista de la Asociación Geológica Argentina XXXI(2): 127-136.

Aschero, C.

1988 Pinturas rupestres, actividades y recursos naturales, un encuadre arqueológico. En Arqueología Contemporánea Argentina, H. Yacobaccio, L. Borrero, L. García, G. Politis, C. Aschero y C. Belelli (eds.), pp. 109-145. Ediciones Búsqueda, Buenos Aires.

Bronk Ramsey, C.

2001 Development of the radiocarbon calibration program OxCal. Radiocarbon 43(2A): 355363.

Cohen, M.

2011 Prácticas Sociales, Estrategias de Visibilidad y Construcción de la Cartografía Social durante el Lapso ca. 1000-1500 AD en Antofagasta de la Sierra, Catamarca. Perspectivas desde el Sitio Peñas Coloradas 3 Cumbre. Tesis de doctorado. Facultad de Filosofía y Letras, Universidad de Buenos Aires.

2014 Miradas desde y hacia lo lugares del poder. Antofagasta de la Sierra entre el 1000 y 1500 D.C. Arqueología 20(1): 47-72.

Cremonte, $\mathrm{M}$.

1991 La tecnología cerámica y las evidencias sobre el origen de los Mitmaqkuna. Anthropologica 9: 237-243.

Cremonte, M. y L. Pereyra Domingorena

2013 Atlas de Pastas Cerámicas Arqueológicas. Petrografía de Estilos Alfareros del NOA. Universidad Nacional de Jujuy, San Salvador de Jujuy.

Cremonte, M. y M. Bugliani

2006-2009 Pasta, forma e iconografía. Estrategias para el estudio de la cerámica arqueológica. Xama 19-23: 239-262.

Courtois, L.

1976 Examen au Microscope Pétrographique des Céramiques Archéologiques. Notes et Monographies Techniques 8. Centre National de la Recherche Scientifique (CNRS), Paris. 
Elías, A.

2010 Estrategias Tecnológicas y Variabilidad de los Conjuntos Líticos de las Sociedades Tardías en Antofagasta de la Sierra (Provincia de Catamarca, Puna Meridional Argentina). Tesis de doctorado. Facultad de Filosofía y Letras, Universidad de Buenos Aires. 2014 Técnicas líticas diversas entre las sociedades de Antofagasta de la Sierra (Provincia de Catamarca, Puna Meridional Argentina) posteriores a ca. 1100 a.p. Estudios Atacameños 47: 59-82.

Escola, P.; López Campeny, S.; Martel, A.; Romano, A.; Hocsman, S. y C. Somonte 2013 Re-conociendo un paisaje. Prospecciones en la quebrada de Miriguaca (Antofagasta de la Sierra, Catamarca). Andes 24(2): 397-423.

Escola, P.; Elías, A.; Gasparotti, L. y N. Sentinelli

2015 Quebrada del río Miriguaca (Antofagasta de la Sierra, Puna Meridional Argentina): nuevo resultados de recientes prospecciones. Intersecciones en Antropología 16(2): 383-396.

Freestone, I.

1991 Extending ceramic petrology. En Recent Developments in Ceramic Petrology, A. Middleton e I. Freestone (eds.), pp. 349-410. British Museum, London.

Gasparotti, L.

2012 Tecnología Cerámica y Producción Alfarera durante el Período Tardío en Antofagasta de la Sierra (Prov. de Catamarca). Tesis de licenciatura. Escuela de Arqueología, Universidad Nacional de Catamarca.

2015 Tecnología cerámica durante el Período Tardío en Antofagasta de la Sierra (Prov. de Catamarca). Una visión a través de cadenas operativas. Cuadernos de la Facultad de Humanidades y Ciencias Sociales 48: 127-142.

Gasparotti, L. y P. Escola

2012 Elecciones tecnológicas en la alfarería. Abordaje preliminar de la cerámica de Corral Alto, Antofagasta de la Sierra (Catamarca). La Zaranda de Ideas 8(1): 49-64.

Gasparotti L. y M. Haros

2015 Contenedores y contenidos. Explorando el consumo de vegetales durante el Período Tardío (Antofagasta de la Sierra, Provincia de Catamarca). Revista del Museo de Antropología 8(2): 47-62. 
González, O.

1992 Geología de la Puna Austral entre los $25^{\circ} 15^{\prime}$ a $26^{\circ} 30^{\prime}$ de latitud Sur y los $66^{\circ} 25^{\prime}$ a $68^{\circ}$ 00’ de longitud Oeste, provincias de Catamarca y Salta, Argentina. Acta Geológica Lilloana XVII(2): 63-88.

Grana, L.

2013 Arqueología y Paleoambiente: Dinámica Cultural y Cambio Ambiental en Sociedades Complejas de la Puna Meridional Argentina. Tesis de doctorado. Facultad de Filosofía y Letras, Universidad de Buenos Aires.

Grant, J.

2014 Manejo Económico de Camélidos en Antofagasta de la Sierra (Puna Meridional Argentina): Una Aproximación Zooarqueológica e Isotópica. Tesis de doctorado. Facultad de Filosofía y Letras, Universidad de Buenos Aires.

Grant, J. y P. Escola

2015 La persistencia de un modo de producción doméstico durante el período Tardío: el caso de Corral Alto (Antofagasta de la Sierra, Argentina). Estudios Atacameños 51: 99-121.

Hammer, Ø.; Harper, D. y P. Ryan

2001 PAST: Paleontological statistics software package for education and data analysis. Palaeontologia Electronica 4(1): 9.

Hodder, I.

2012 Entangled: An Archaeology of the Relationships Between Humans and Things. WileyBlackwell, Chichester.

Ingold, $\mathrm{T}$.

2007 Material against materiality. Archaeological Dialogues 14(1): 1-16.

Lemonnier, $\mathrm{P}$.

1992 Elements for an Anthropology of Technology. Museum of Anthropology, Michigan.

1993 Introduction. En Technological Choices: Transformation in Material Cultures since the Neolithic, P. Lemonnier (ed.), pp. 1-35. Routledge, London.

Martel A. y C. Aschero

2007 Pastores en acción: imposición iconográfica vs. autonomía temática. En Producción y Circulación Prehispánicas de Bienes en el Sur Andino, A. Nielsen, M. Rivolta, V. Seldes, M. Vázquez y P. Mercolli (eds.), pp. 329-349. Editorial Brujas, Córdoba. 
Murra, J

1975 El Control Vertical de un Máximo de Pisos Ecológicos en la Economía de las Sociedades Andinas. Formaciones Económicas y Políticas del Mundo Andino. Instituto Estudios Peruanos, Lima.

Nielsen, A.

2006 Plazas para los antepasados: Descentralización y poder corporativo en las formaciones políticas preinkaicas de los Andes circumpuneños Estudios Atacameños 31: 63-89.

Núñez Regueiro, V.

1974 Conceptos instrumentales y marco teórico en relación al análisis del desarrollo cultural del Noroeste argentino. Revista del Instituto de Antropología V: 169-180.

Olivera, D.

1992 Tecnología y Estrategias de Adaptación en el Formativo (Agro-alfarero Temprano) de la Puna Meridional Argentina. Un Caso de Estudio: Antofagasta de la Sierra (Pcia. de Catamarca, R.A.). Tesis de doctorado. Facultad de Ciencias Naturales y Museo, Universidad Nacional de La Plata.

Olivera, D. y S. Vigliani

2000-2002 Proceso cultural, uso del espacio y producción agrícola en la Puna Meridional Argentina. Cuadernos del INAPL 19: 459-481.

Olivera, D.; Elías, A.; Salminci, P.; Tchilinguirian, P.; Grana, L.; Grant, J. y P. Miranda 2008 Nuevas evidencias del proceso sociocultural en Antofagasta de la Sierra. Informe de campaña año 2007. La Zaranda de Ideas 4: 119-140.

Olivera, D.; Tchilinguirian, P. y L. Grana 2004 Paleoambiente y arqueología en la Puna Meridional Argentina: archivos ambientales, escalas de análisis y registro arqueológico. Relaciones de la Sociedad Argentina de Antropología XXIX: 229-247.

Olivera, D.; Vigliani, S.; Elías, A.; Grana, L. y P. Tchilinguirian 2003-2005 La ocupación Tardío-Inka en la Puna meridional: el sitio Campo Cortaderas. Cuadernos del INAPL 20: 257-277. 
Páez, M.

2010 Tecnología Alfarera del Último Milenio de Ocupación Aborigen del Valle de Tafí (Prov. de Tucumán). Tesis de doctorado. Facultad de Ciencias Naturales y Museo, Universidad Nacional de La Plata.

Palamarczuk, V.

2008 Un análisis de la cerámica arqueológica de cuatro sitios en el bajo de Rincón Chico. En Estudios Arqueológicos en Yocavil, M. Tarragó y L. González (eds.), pp. 20-80. Asociación de Amigos del Museo Etnográfico, Buenos Aires.

Pérez M. y L. Gasparotti

2016 Caracterización petrográfica de las pastas cerámicas de Antofagasta de la Sierra (Puna Austral Argentina). Un enfoque comparativo a nivel intersitios. Comechingonia 20(1): 175202.

Pérez, M.

2013 Investigación sobre el Período Tardío-Inca en las Localidades Arqueológicas de Antofagasta de la Sierra (Puna Sur) y Cuenca del Río Doncellas (Puna Norte): Una Aproximación a través de la Cerámica. Tesis de doctorado. Facultad de Filosofía y Letras, Universidad de Buenos Aires.

Puente, V.

2010 Prácticas de Producción Alfarera en el Valle del Bolsón (Belén, Catamarca). Materias Primas y Modos de Hacer ca. 900-1600 D.C. Tesis de doctorado. Facultad de Filosofía y Letras, Universidad de Buenos Aires.

2015 Relaciones de interacción entre Antofagasta de la Sierra y el valle del Bolsón (Catamarca, Argentina). Primeros aportes desde la alfarería ca. 900-1600 D.C. Chungara 47(3): 369-385.

Raffino, R. y E. Cigliano

1973 “La Alumbrera” -Antofagasta de la Sierra-. Un modelo de ecología cultural prehispánica. Relaciones de la Sociedad Argentina de Antropología VII: 241-258.

Tarragó, M.

2000 Chakras y pukara. Desarrollos sociales tardíos. En Los Pueblos Originarios y la Conquista. Nueva Historia Argentina, M. Tarragó (ed.), vol. 1, pp. 257-300. Editorial Sudamericana, Buenos Aires. 
Tchilinguirian, P. y D. Olivera

2000 Aportes edafológicos para el estudio de campos agrícolas prehispánicos Bajo del Coypar, provincia de Catamarca. Cuaternario y Ciencias Ambientales Publicación Especial 4: 51-56.

2010 Agricultura, ambiente y sustentabilidad agrícola en el desierto: el caso Antofagasta de la Sierra (Puna Argentina, 26 S). En Arqueología de la Agricultura: Casos de Estudio en la Región Andina Argentina, A. Korstanje y M. Quesada (eds.), pp. 102-127. Ediciones Magna, San Miguel de Tucumán.

Vigliani, S.

1999 Cerámica y Asentamiento: Sistema de Producción Agrícola Belén-Inka. Tesis de licenciatura. Facultad de Filosofía y Letras, Universidad de Buenos Aires.

Wynveldt, F. y M. Flores

2014 La obsidiana en el paisaje Tardío del Valle de Hualfín (Depto. de Belén. Prov. de Catamarca). Arqueología 20 Dossier: 193-216. 